\title{
Application of UV and IR radiation for spatial characterization of Bragg gratings
}

\author{
I. G. Korolev, S. A. Vasiliev, O. I. Medvedkov, E. M. Dianov \\ Fiber Optics Research Center at the General Physics Institute of the Russian Academy of Sciences \\ 38, Vavilov Str., 117756, Moscow, Russia, fax: (095) 135-8139 \\ ivan@fo.gpi.ru \\ F. Knappe, Ch. Knothe, H. Renner, E. Brinkmeyer \\ Technische Universität Hamburg-Harburg \\ Eißendorfer Str. 40, 21073 Hamburg, Germany, Tel.: +49-40-42878-3437 \\ Knappe@tu-harburg.de
}

\begin{abstract}
OSDR techniques for spatial characterization of fiber Bragg gratings based on local phase induction by IR or UV radiation have been developed. Both radiation types provide longitudinally and cross-sectionally uniform fiber excitation, high spatial resolution and good sensitivity of measurements.

(C)2001 Optical Society of America

OCIS codes: (060.2300) Fiber measurements; (060.2340) Fiber optics components
\end{abstract}

\section{Introduction}

Fiber Bragg gratings (FBGs) are widely used for telecommunication and sensor systems. In some cases it is important to know the axial distribution of the grating parameters as well as the exact grating location in the fiber.

The most common and simplest way of the grating characterization is measuring its transmission/reflection spectrum. This method determines only the integral characteristics of the FBG. To obtain the spatial distribution of local grating properties other techniques such as OLCR [1], side-diffraction [2], heat-scan [3] can be used.

An optical space domain reflectometry (OSDR) method for obtaining the spatial variation of the complex coupling coefficient $\kappa(z)$ of FBG with high spatial resolution (about $100 \mu \mathrm{m}$ ) has been proposed in [4]. In this paper a He-Ne laser has been used for local introducing a small optical phase perturbation in the grating structure. Because of the small absorption of this radiation type by silica glass, the tested grating had to be covered with an absorbing layer, typically with limited longitudinal homogeneity.

In the present work we suggest application of more suitable laser sources (a CO-laser and a frequency-doubled Ar-ion laser) for local induction of a phase perturbation in the OSDR technique.

\section{Experiments}

The OSDR technique for spatial characterization of grating properties is described in [4]. It is based on introducing a local AC phase perturbation scanned along the grating and measuring the transmission variations at a fixed wavelength outside the stop band. In our present experiments we used a similar setup with either a frequencydoubled Ar-ion laser $(\lambda=244 \mathrm{~nm})$ or a CO-laser $(\lambda \approx 5 \mu \mathrm{m})$ instead of a He-Ne laser. The penetration depth of COlaser radiation into silica glass is $100-200 \mu \mathrm{m}$ which allows us to heat the fiber cross-section with good uniformity. The UV-light of the Ar-ion laser induces a transient optical phase perturbation directly in the fiber core [5]. In both cases no additional absorbing coating is required and the spatial resolution and uniformity of phase changes are improved. In addition these radiation types can induce a rather large phase which increases the sensitivity of the method.

Highly reflecting Bragg gratings written in hydrogen loaded single mode fiber (SMF-28) have been tested in our experiments. The gratings were written in a Lloyd interferometer scheme by radiation of frequency-doubled Ar-ion laser and had a length of about $5 \mathrm{~mm}$.

It should be noted that the radiation types we used can change the refractive index distribution. The CO-laser radiation at high power can heat the fiber strongly which can lead to annealing of the induced index, whereas UV light can induce an additional average index change. Therefore the irradiation dose, which depends on the irradiation density and the scan speed, should be small to avoid the grating degradation. On the other hand, these effects could be used intentionally for modification of the grating structure.

During the grating testing we used UV and IR power densities that did not lead to perceptible permanent changes of the grating overall spectrum. For the UV-laser, the power density was $\sim 30 \mathrm{~W} / \mathrm{cm}^{2}$, that resulted in cumulated 
UV-dose of about $700 \mathrm{~J} / \mathrm{cm}^{2}$ per scan. This dose corresponds to induced permanent index changes of about $\Delta n_{\text {ind }} \sim$ $2 \cdot 10^{-5}$, which is relatively small compared with an average index induced in the fiber during the grating preparation $\Delta n_{\text {avr }} \sim 10^{-3}$. In the case of the CO-laser irradiation the introduced phase shift provided measurable change of the grating transmission spectrum and was estimated as $0.14 \mathrm{rad}$. This phase value corresponds to a heating temperature of about $30^{\circ} \mathrm{C}$ which is much less than the annealing temperature of the UV-induced index. Thus for weak Bragg gratings with small $\Delta n_{\text {avr }}$ of the order of $10^{-4}$ application of CO-laser irradiation seems to be more appropriate.

Both the UV and the IR laser beams used for phase perturbation were chopped with a frequency of $\sim 230 \mathrm{~Hz}$ and the OSDR signal was detected by a lock-in amplifier.

\section{Results and discussion}

An OSDR trace measured for a detuning $\Delta \lambda \approx 1.5 \mathrm{~nm}$ of the DFB test laser wavelength from the grating central wavelength is plotted in Fig.1a. The grating position corresponds to the region between 1 and $6 \mathrm{~mm}$ in the trace where the signal oscillations are clearly visible. The number of OSDR oscillations in the trace is defined by the detuning and was selected in order to be suitable for qualitative processing of the measured OSDR signal.
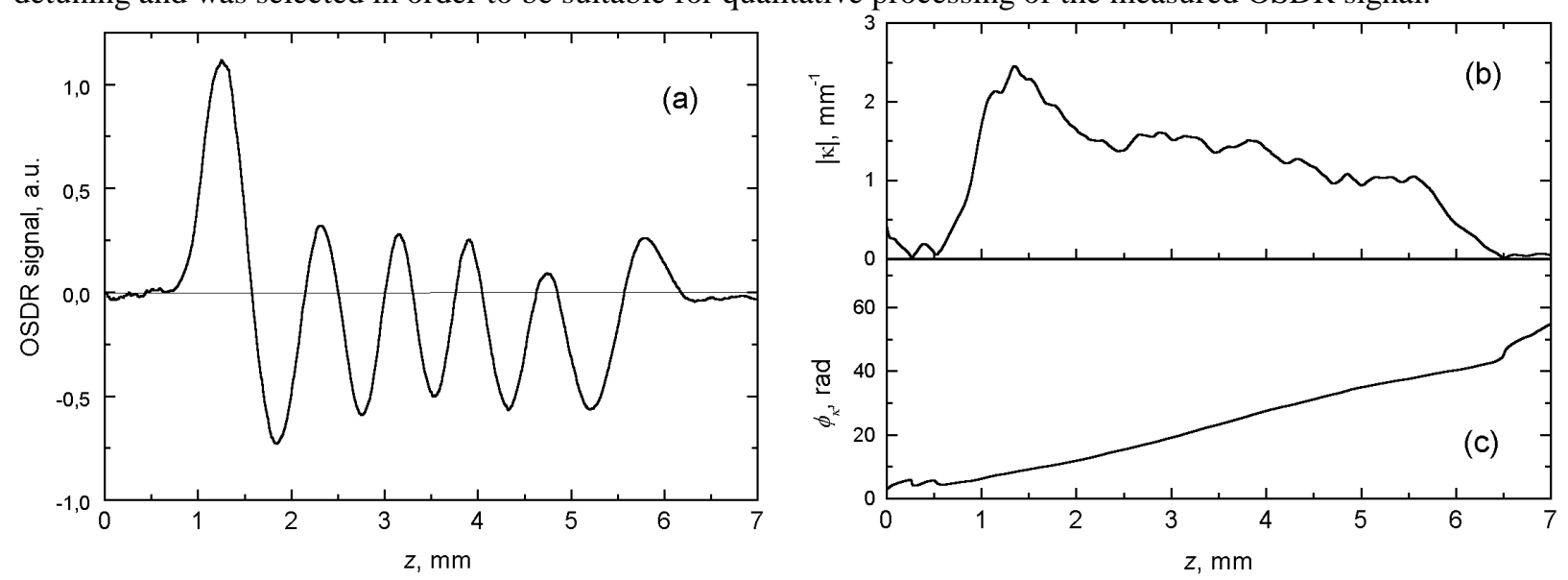

Fig.1. OSDR-signal measured for CO-laser light excitation (a); amplitude (b) and phase (c) of the grating coupling coefficient calculated from the OSDR-data.

The axial distributions of the amplitude $|\kappa(z)|$ and the phase $\phi_{k}(z)$ of the coupling coefficient of the tested FBG calculated from the OSDR signal are presented in Fig.1b and 1c, respectively. The amplitude of the coupling coefficient is related to the amplitude of the refractive index modulation as $|\kappa(z)|=\pi \eta \Delta n_{\bmod }(z) / \lambda_{0}$, where $\eta$ is the fundamental mode portion propagating in the fiber core. The latter relation allows one to obtain $\Delta n_{\bmod }(z)$.

Fig.2a shows the axial distribution of the induced index modulation measured by different methods (OSDR techniques with IR and UV excitation as well as with the side-diffraction technique using a He-Ne laser). Coincidence within 10-15\% deviation between these curves has been achieved.

The spatial derivative of the phase is related to the grating parameters as

$$
\frac{d \phi_{\kappa}(z)}{d z} \approx 2 \pi\left[2 n_{e f f}^{0} \frac{\Delta \lambda}{\lambda_{0}^{2}}+\frac{2 \Delta n_{e f f}(z)}{\lambda_{0}}+\frac{\Delta \Lambda(z)}{\Lambda_{a v r}^{2}}\right],
$$

where $\lambda_{0}=2 n_{\text {eff }}^{0} \Lambda_{\text {avr }}$ is the Bragg resonance wavelength, $\Delta n_{\text {eff }}(z)=n_{\text {eff }}(z)-n_{\text {eff }}^{0}$ is the deviation of the local effective index from that of the fundamental mode $n_{\text {eff }}^{0}$, and $\Delta \Lambda(z)=\Lambda(z)-\Lambda_{a v r}$ is the grating period deviation from the average period $\Lambda_{\text {avr }}$. The first term in equation (1) is defined by the wavelength detuning $\Delta \lambda$ and is constant. The second and the third terms are determined by the FBG properties and cannot be obtained independently. Both of them can give a Bragg wavelength shift and special suggestions or experiments should be performed in order to separate these grating parameters. It seems reasonable to assume a high contrast in the index modulation and the pitch profile to be close to a sinusoidal one. This assumption gives $\Delta n_{\text {avr }}(z)=\Delta n_{\bmod }(z)$, where $\Delta n_{\text {avr }}(z)$ is defined through $\Delta n_{\text {eff }}(z)=\eta \Delta n_{\text {avr }}(z)$. Using this assumption and (1) the relative variation of the grating period has been obtained and presented in Fig.2b. With the data obtained for the coupling coefficient we calculated the transmission spectra of the grating. Using only the index modulation distribution and assuming the 
grating period to be constant we found a poor coincidence between the calculated and the measured spectra (Fig.3, dotted and solid lines, respectively). Taking into account both the period variation and the index modulation distributions, however, gave us a spectrum (Fig.3, dashed line) close to the measured one (Fig.3, solid line).
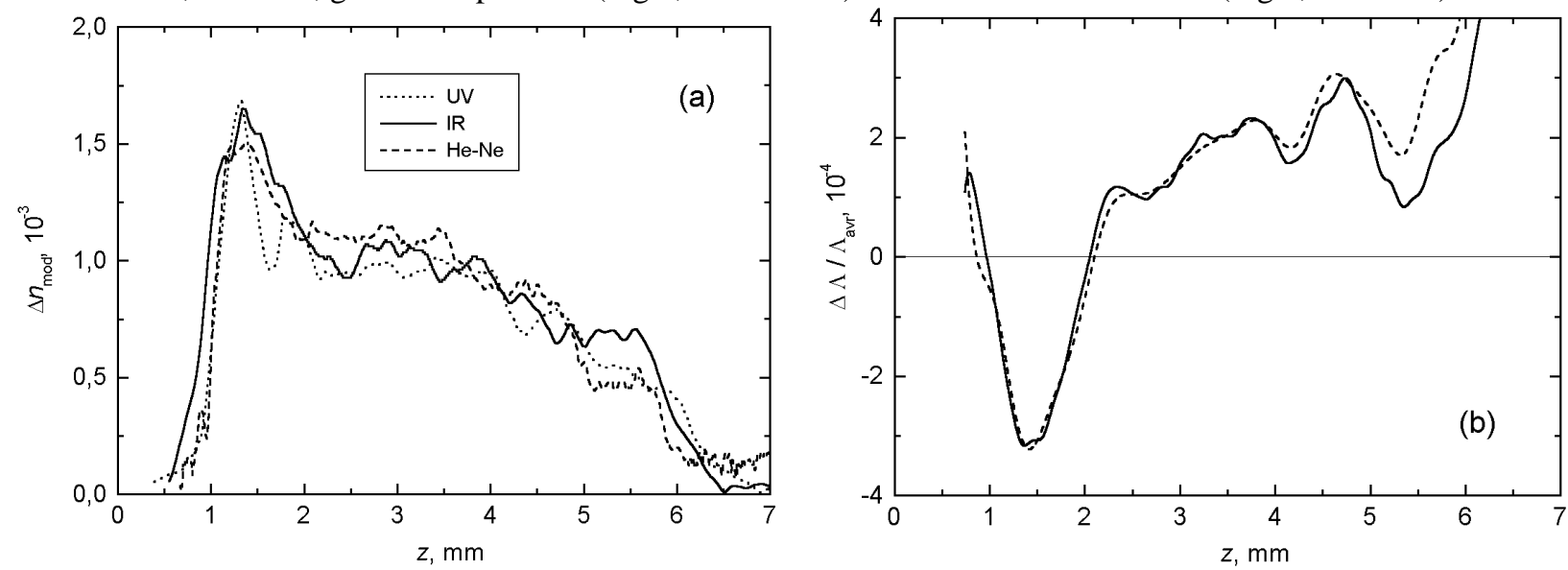

Fig.2. Calculated distributions of index modulation amplitude measured by UV- and IR-OSDR techniques as well as by side-diffraction technique(a); relative period distribution for two different IR measurements (b).

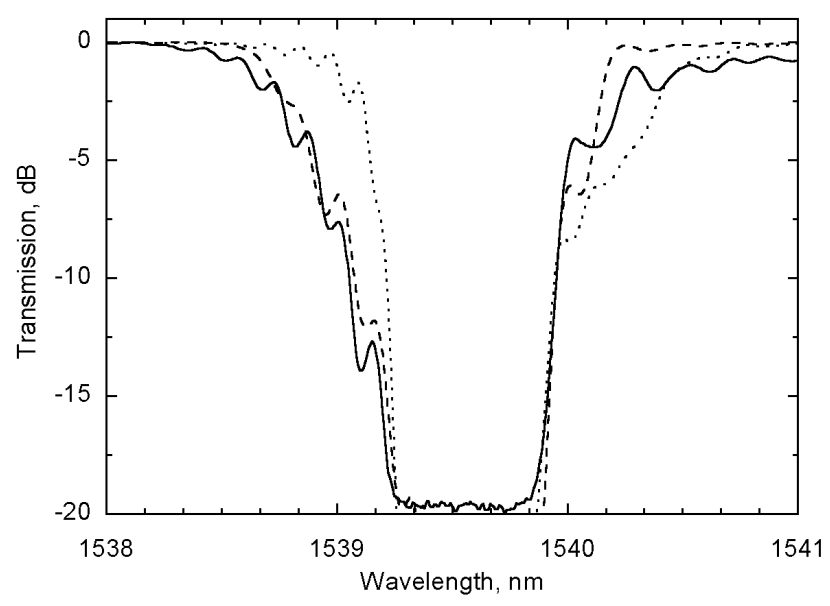

Fig.3. The measured FBG transmission spectrum (solid line), the spectra calculated taking into account measured $\Delta n_{\bmod }(z)$ (dotted line) and both $\Delta n_{\bmod }(z)$ and $\Delta \Lambda(z)$ (dashed line)

\section{Conclusions}

Optical space domain reflectometry techniques based on IR (CO-laser) and UV (frequency-doubled Ar-ion laser) local irradiation of fiber Bragg gratings have been developed. These methods do not require an additional covering the grating with an absorbing layer, and have improved sensitivity, uniformity and spatial resolution. Comparison of obtained $\Delta n_{\text {mod }}$ distributions with that measured by the side-diffraction technique showed good coincidence within $10-15 \%$ deviation. Grating phase information provided by the OSDR technique allowed us to achieve satisfactory agreement between calculated and measured grating spectra.

\section{References}

1. P. Lambelet, P.Y. Fonjallaz, H.G. Limberger, R.P. Salathé, C. Zimmer, H.H. Gilgen, "Bragg grating characterization by Optical LowCoherence Reflectometry", IEEE Photon. Techn. Lett. 5, 565-567 (1993).

2. P.A. Krug, R. Stolte, R. Ulrich, "Measurement of index modulation along an optical fiber Bragg grating", Opt. Lett., 20, 1767-1769 (1995).

3. N.Roussel, S.Magne, C.Martinez, P.Ferdinand, "Measurement of index modulation along fiber gratings by side scattering and local heating techniques", Opt. Fib. Techn. 5, 119-132, (1999).

4. E.Brinkmeyer, G.Stolze, D.Johlen, "Optical space domain reflectometry (OSDR) for determination of strength and chirp distribution along optical fiber gratings", in Bragg Gratings, Photosensitivity, and Poling in Glass Fibers and Waveguides: Applications and Fundamentals, Vol.17 of OSA Techn. Dig. Series (Optical Society of America, Washington, D.C., 1997), pp.33-35.

5. M.B. Danailov, T. Gasmi, P. Apai, "Transient refraction index changes in UV-exposed optical fibres”, Electr. Lett. 32, 482-483, (1996). 